\title{
Synchronization of a Fractional-order Complex System
}

\author{
Honggang Dang ${ }^{\mathrm{a}}$, Xiaoya Yang, XiaoJun Liu \\ School of Mathematics and statistics, Tianshui Normal University, Tianshui, China \\ afly0164@126.com
}

Keywords: Fractional-order system, synchronization, chaos.

\begin{abstract}
In this paper, synchronization of a fractional-order complex system is studied. Based on the stability theory of fractional-order systems, the scheme of synchronization for the fractional-order complex system is proposed. the synchronization for the system is realized by designing appropriate controllers. Numerical simulations are used to demonstrate the effectiveness of the proposed scheme.
\end{abstract}

\section{Introduction}

Recently, chaos synchronization has attracted increasing interests which was proposed in 1990 [1]. Synchronization of integer-order systems has been studied extensively and several methods are extended to synchronize fractional-order complex systems [2-4]. As well known, fractional calculus is very important in mathematical modeling. As research continues, the importance of fractional-order systems with complex variables is realized by many researchers, which can be widely used to describe a variety of physical phenomena.

\section{The fractional-order complex system}

In [5], a new three-dimensional system was presented, which can be described by the following differential equations

$$
\left\{\begin{array}{l}
\dot{y}_{1}=a\left(y_{2}-y_{1}\right) \\
\dot{y}_{2}=y_{1} y_{3}-y_{2} \\
\dot{y}_{3}=b-y_{1} y_{2}-c y_{3}
\end{array},\right.
$$

where $y=\left(y_{1}, y_{2}, y_{3}\right)^{\mathrm{T}}$ is the state variable vector of the system, $a, b, c$ are parameters. When the parameters $a=5, b=16, c=1$, the system exists an chaotic attractor.

In here, the state variables of system (1) are defined in the complex field, then the corresponding fractional-order system is defined as

$$
\left\{\begin{array}{l}
D^{q} y_{1}=a\left(y_{2}-y_{1}\right) \\
D^{q} y_{2}=y_{1} y_{3}-y_{2} \\
D^{q} y_{3}=b-\frac{1}{2}\left(\bar{y}_{1} y_{2}+y_{1} \bar{y}_{2}\right)-c y_{3}
\end{array},\right.
$$

where $q$ is the order of derivative, $y=\left(y_{1}, y_{2}, y_{3}\right)^{\mathrm{T}}$ is the vector of state variables. $y_{1}=x_{1}+i x_{2}, y_{2}=x_{3}+i x_{4}$ are complex variables, $y_{3}=x_{5}$ is real variable, and $i=\sqrt{-1}$. Then the complex variables in the system are separated into the real and imaginary parts, respectively. According to the linearity of the Caputo differential operator, the system (2) can be rewritten as 


$$
\left\{\begin{array}{l}
D^{q} x_{1}=a\left(x_{3}-x_{1}\right) \\
D^{q} x_{2}=a\left(x_{4}-x_{2}\right) \\
D^{q} x_{3}=x_{1} x_{5}-x_{3} \\
D^{q} x_{4}=x_{2} x_{5}-x_{4} \\
D^{q} x_{5}=b-\left(x_{1} x_{3}+x_{2} x_{4}\right)-c x_{5}
\end{array}\right.
$$

Compared with the system (2), (3) is more convenient for analysis and numerical simulation. By numerical computation, the maximum Lyapunov exponent of the system (3) with $a=5, b=16, c=1, q=0.99$ is $\lambda_{1}=0.071$, which means the system (3) is chaotic. The chaotic attractors on different phase space projections are shown in Fig.1

(a)

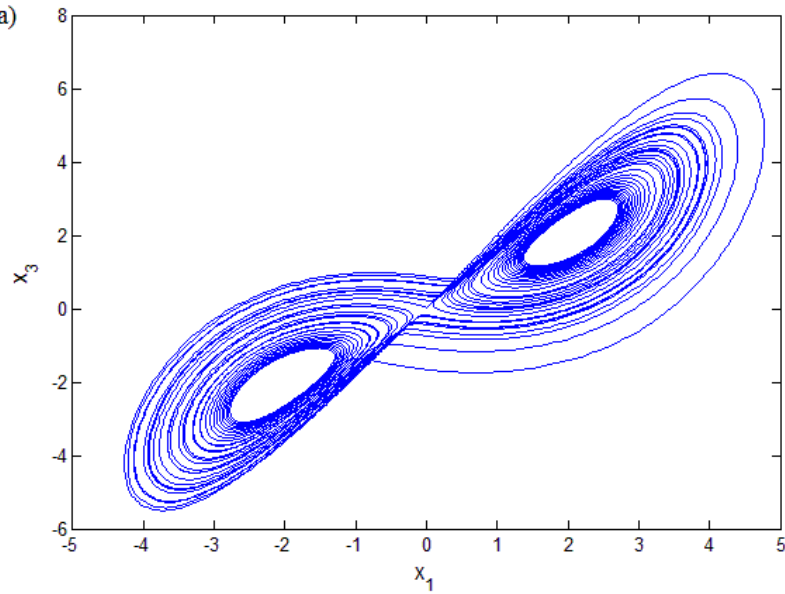

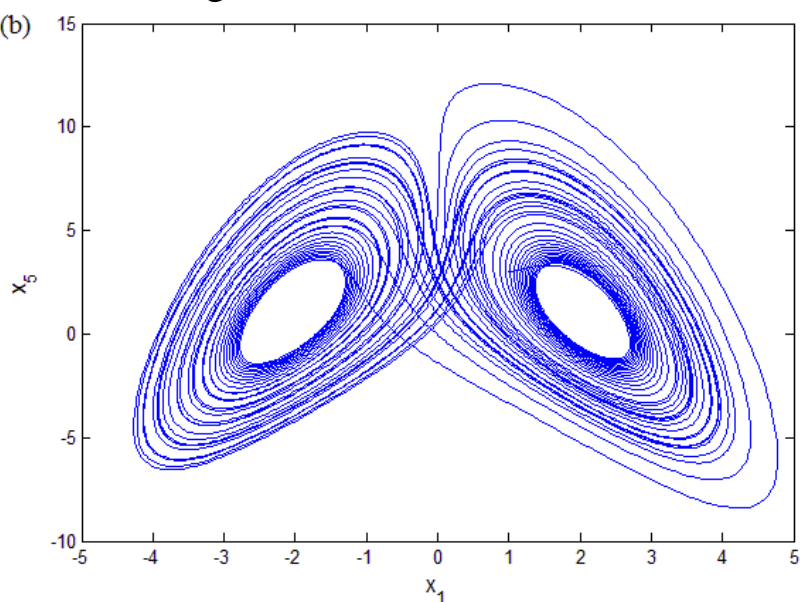

Fig.1. The chaotic attractors of the system (3) projected onto different phase plane. (a) $x_{1}-x_{3}$ phase plane; (b) $x_{1}-x_{5}$ phase plane.

In this section, the synchronization for the fractional-order complex system (3) will be studied.

\section{Synchronization}

Firstly, the synchronization scheme for a fractional-order complex system is introduced. The drive and response systems are follows, respectively:

$$
\begin{aligned}
& D^{\alpha} y=f(y), \\
& \quad D^{\beta} Z=g(z)+u(y, z),
\end{aligned}
$$

where $\alpha \in(0,1)$ and $\beta \in(0,1)$ are the derivative orders of systems (4) and (5), respectively. The complex variables are defined as $y_{j}=x_{2 j-1}+i x_{2 j} \quad, \quad z_{j}=x_{2 j-1}^{\prime}+i x_{2 j}^{\prime},(j=1,2, \cdots, n)$. $u(y, z): R^{n} \times R^{n} \rightarrow R^{n}$ is a synchronization controller which will be designed later. $e=z-y$ is defined as the error vector, synchronization of systems (4) and (5) is achieved if the following condition is satisfied

$$
\lim _{t \rightarrow+\infty}\|e\|=\lim _{t \rightarrow+\infty}\|z-y\|=0,
$$

where $\|\cdot\|$ is the Euclidean norm. The error vector can be rewritten as $e=e^{\text {real }}+i e^{\text {image }}$, where $e^{\text {real }}=z^{\text {real }}-y^{\text {real }}, e^{\text {image }}=z^{\text {image }}-y^{\text {image }}$. It is need to define a compensation controller $\theta(y)=D^{\beta}(y)-g(y)$ and a synchronization controller $u(y, z)=\theta(y)+\tau(y, z)$ for system (5). Then, we can get the error dynamical system by substituting the controllers into the response system (5) as following:

$$
D^{\beta} e=g(z)-g(y)+\tau(y, z)=A(y, z) e++\tau(y, z) .
$$

If $\tau(y, z)$ is chosen as $B(y, z) e$, and system (7) is rewritten as 


$$
D^{\beta} e=A(y, z) e+B(y, z) e=[A(y, z)+B(y, z)] e .
$$

then (8) is asymptotically converge to zero when error vector tends to zero when $t \rightarrow+\infty$.

The system (3) is taken as the drive system, the corresponding response system with controller is

$$
\left[\begin{array}{l}
D^{\beta} z_{1} \\
D^{\beta} z_{2} \\
D^{\beta} z_{3}
\end{array}\right]=\left[\begin{array}{l}
a\left(z_{2}-z_{1}\right) \\
z_{1} z_{3}-z_{1} \\
b-\frac{1}{2}\left(\bar{z}_{1} z_{2}+z_{1} \bar{z}_{2}\right)-c z_{3}
\end{array}\right]+u(y, z),
$$

$z_{1}=x_{1}^{\prime}+i x_{2}^{\prime}, z_{2}=x_{3}^{\prime}+i x_{4}^{\prime}$ are complex variables, $z_{3}=x_{5}^{\prime}$ is real variable. The system (9) can be expressed as

$$
\left[\begin{array}{l}
D^{\beta} x_{1}^{\prime} \\
D^{\beta} x_{2}^{\prime} \\
D^{\beta} x_{3}^{\prime} \\
D^{\beta} x_{4}^{\prime} \\
D^{\beta} x_{5}^{\prime}
\end{array}\right]=\left[\begin{array}{l}
a\left(x_{3}^{\prime}-x_{1}^{\prime}\right) \\
a\left(x_{4}^{\prime}-x_{2}^{\prime}\right) \\
x_{1}^{\prime} x_{5}^{\prime}-x_{1}^{\prime} \\
x_{2}^{\prime} x_{5}^{\prime}-x_{2}^{\prime} \\
b-\left(x_{1}^{\prime} x_{3}^{\prime}+x_{2}^{\prime} x_{4}^{\prime}\right)-c x_{5}^{\prime}
\end{array}\right]+u(y, z)
$$

By computation, we can get $\mathrm{A}\left(x_{j}, x_{j}^{\prime}\right)$ is following

$$
\mathrm{A}\left(x_{j}, x_{j}^{\prime}\right)=\left[\begin{array}{ccccc}
-a & 0 & a & 0 & 0 \\
0 & -a & 0 & a & 0 \\
x_{5}^{\prime} & 0 & -1 & 0 & x_{1} \\
0 & x_{5}^{\prime} & 0 & -1 & x_{2} \\
-x_{3}^{\prime} & -x_{4}^{\prime} & -x_{1} & -x_{2} & -c
\end{array}\right] .
$$

Meanwhile, the matrix $\mathrm{B}\left(x_{j}, x_{j}^{\prime}\right)$ is

Therefore, the error dynamical system is

$$
\mathrm{B}\left(x_{j}, x_{j}^{\prime}\right)=\left[\begin{array}{ccrrc}
0 & 0 & 0 & 0 & x_{3}^{\prime} \\
0 & 0 & 0 & 0 & x_{4}^{\prime} \\
-x_{5}^{\prime}-a & 0 & 0 & 0 & 0 \\
0 & -x_{5}^{\prime}-a & 0 & 0 & 0 \\
0 & 0 & 0 & 0 & 0
\end{array}\right] .
$$

$$
\left[\begin{array}{l}
D^{\beta} e_{1} \\
D^{\beta} e_{2} \\
D^{\beta} e_{3} \\
D^{\beta} e_{4} \\
D^{\beta} e_{5}
\end{array}\right]=\left[\begin{array}{ccccc}
-a & 0 & a & 0 & x_{3}^{\prime} \\
0 & -a & 0 & a & x_{4}^{\prime} \\
-a & 0 & -1 & 0 & x_{1} \\
0 & -a & 0 & -1 & x_{2} \\
-x_{3}^{\prime} & -x_{4}^{\prime} & -x_{1} & -x_{2} & -c
\end{array}\right]\left[\begin{array}{c}
e_{1} \\
e_{2} \\
e_{3} \\
e_{4} \\
e_{5}
\end{array}\right] .
$$

The orders of derivative are $q=\beta=0.99$. The synchronization of simulation for the drive and response systems are showed in Fig.2, from which it can be seen that the error variables $e_{1}, e_{2}, e_{3}, e_{4}$, and $e_{5}$ converge to zero, which means the synchronization between the systems (3) and (10) is realized under the controllers. 


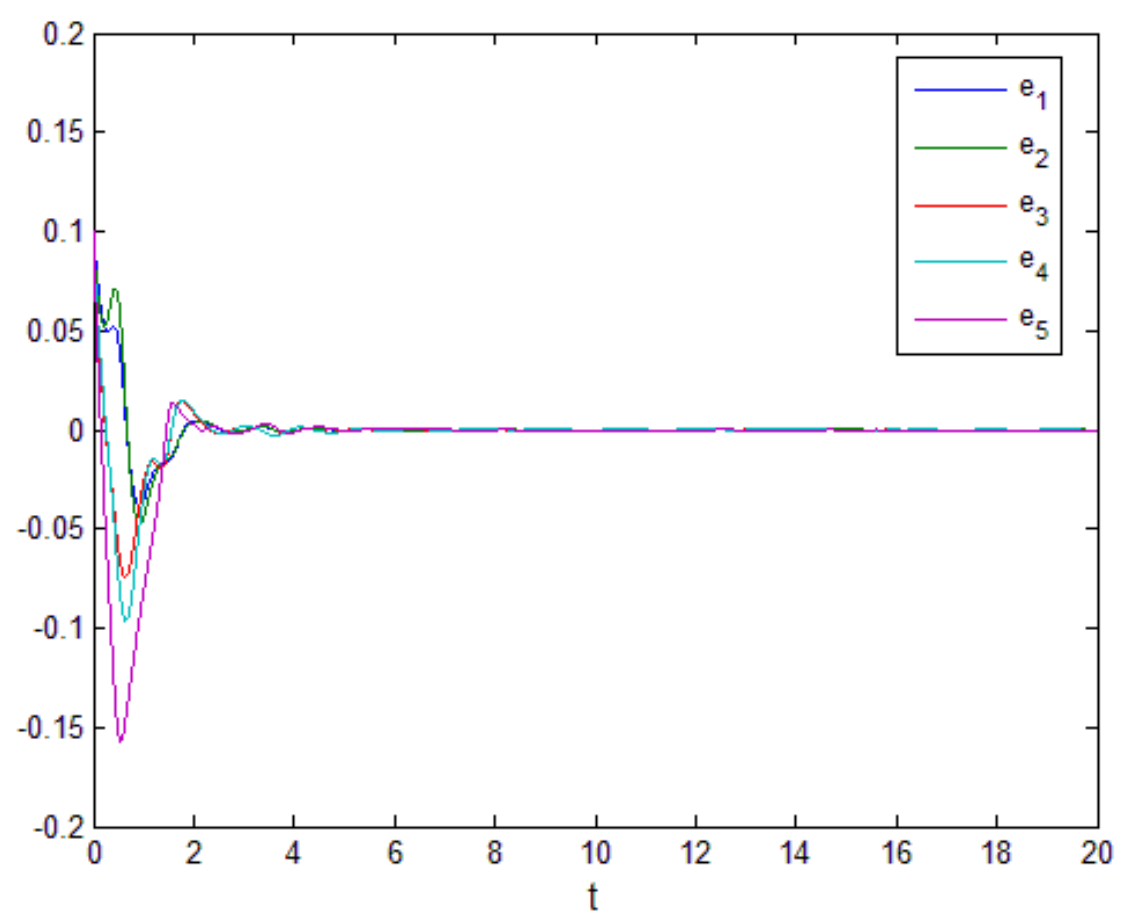

Fig.2. synchronization errors of the systems (3) and (10)

\section{Conclusions}

In this paper, synchronization of a fractional-order complex system is studied. Based on the stability theory of fractional-order systems, the scheme of synchronization for the fractional-order complex system is proposed. the synchronization for the system is realized by designing appropriate controllers. Numerical simulations are used to demonstrate the effectiveness of the proposed scheme.

\section{References}

[1] L.M. Pecora, and T.L. Carroll: Synchronization in chaotic systems. Physical Review Lette, 64(1990): 821-841.

[2] G.M. Mahmoud, T. Bountis, G.M. Abdel-Latif, and E. E. Mahmoud: Chaos synchronization of two different chaotic complex Chen and Lü systems. Nonlinear Dynamics, 55(2009): 43-53.

[3] G.M. Mahmoud, and E.E. Mahmoud: Complete synchronization of chaotic complex nonlinear systems with uncertain parameters. Nonlinear Dynamics, 62(2010): 875-882.

[4] G.M. Mahmoud, and E.E. Mahmoud: Synchronization and control of hyperchaotic complex Lorenz system. Mathematics and Computers in Simulation, 80(2010)2286-2296.

[5] X.F. Li, Y.D. Chu, J.G. Zhang, and Y.X. Chang: Nonlinear dynamics and circuit implementation for a new autonomous chaotic system. Chaos Solitions \& Fractals, 41(2009): 2360-2370. 